\title{
muCool: a next step towards efficient muon beam compression
}

\author{
I. Belosevic ${ }^{1, a}$, A. Antognini ${ }^{1,2}$, Y. Bao ${ }^{2,5}$, A. Eggenberger ${ }^{1}$, M. Hildebrandt ${ }^{2}$, R. Iwai ${ }^{1}$, D. M. Kaplan ${ }^{3}$, \\ K. S. Khaw ${ }^{1,6}$, K. Kirch ${ }^{1,2}$, A. Knecht ${ }^{2}$, A. Papa ${ }^{2,4}$, C. Petitjean ${ }^{2}$, T. J. Phillips ${ }^{3}$, F. M. Piegsa ${ }^{1,7}$, N. Ritjoho ${ }^{2}$, \\ A. Stoykov ${ }^{2}$, D. Taqqu ${ }^{1}$, G. Wichmann ${ }^{1}$ \\ ${ }^{1}$ Institute for Particle Physics and Astrophysics, ETH Zürich, 8093 Zürich, Switzerland \\ ${ }^{2}$ Paul Scherrer Institute, 5232 Villigen-PSI, Switzerland \\ ${ }^{3}$ Illinois Institute of Technology, Chicago, IL 60616, USA \\ ${ }^{4}$ Dipartimento di Fisica, Università di Pisa, and INFN sez. Pisa, Largo B. Pontecorvo 3, 56127 Pisa, Italy \\ 5 Present address: Institute of High Energy Physics, Chinese Academy of Sciences, Beijing 100049, China \\ ${ }^{6}$ Present address: Department of Physics, University of Washington, Seattle, WA 98195, USA \\ ${ }^{7}$ Present address: Laboratory for High Energy Physics, Albert Einstein Center for Fundamental Physics, University of Bern, 3012 Bern, Switzerland
}

Received: 20 November 2018 / Accepted: 9 May 2019 / Published online: 21 May 2019

(c) The Author(s) 2019

\begin{abstract}
A novel device to compress the phase space of a muon beam by a factor of $10^{10}$ with a $10^{-3}$ efficiency is under development. A surface muon beam is stopped in a helium gas target consisting of several compression stages, wherein strong electric and magnetic fields are applied. The spatial extent of the stopped muon swarm is decreased by means of these fields until muons with $\mathrm{eV}$ energy are extracted into vacuum through a small orifice. It was observed that a $20 \mathrm{~cm}$ long muon stop distribution can be compressed in the longitudinal direction to a sub-mm extent within $2 \mu \mathrm{s}$. Additionally, a drift perpendicular to the magnetic field of the compressed lowenergy muon swarm was successfully demonstrated, paving the way towards extraction from the gas and re-acceleration of the muons.
\end{abstract}

\section{Introduction}

Standard surface muon beams have a relatively poor phase space quality. An improvement of the muon beam quality would open the way for new experiments in low energy particle physics, atomic physics and material research. Examples of such experiments include muonium $1 \mathrm{~S}-2 \mathrm{~S}$ spectroscopy, muon electric dipole moment (EDM) measurement and muon spin rotation $(\mu \mathrm{SR})$ applications. Due to the limited lifetime of the muons ( $\tau=2197 \mathrm{~ns}$ ), a fast cooling scheme is required, and thus conventional beam cooling methods such as stochastic [1] or electron cooling [2] cannot be applied. Several alternative muon beam cooling schemes have been developed: based on frictional cooling [3], ioniza-

\footnotetext{
a e-mail: ivanabe@phys.ethz.ch
}

tion cooling (MICE) [4] or moderation in solid rare gas layers [5]. The common principle of the phase space compression obtained using these techniques is momentum spread reduction in all directions by moderation in matter and subsequent re-acceleration in the longitudinal direction.

In contrast, the scheme presented here (muCool device [6]) moderates the muon beam momentum while simultaneously compressing its beam spot. Such a phase space compression is achieved by stopping the incoming $\mu^{+}$in He gas, while manipulating them in the gas with electric and magnetic fields in order to decrease their spatial extent. After compression, which takes place within $10 \mu \mathrm{s}$, the muons are extracted at $\mathrm{eV}$ energies from the $\mathrm{He}$ gas and re-accelerated. When this technique is applied to a standard surface $\mu^{+}$ beam, the $6 \mathrm{D}$ phase space is reduced by a factor of $10^{10}$ with an efficiency of $10^{-3}$, mainly limited by the muon lifetime. Thus, the brightness of the incoming beam is enhanced by a factor of $10^{7}$.

The decrease of the muon swarm extent is obtained by making the drift velocity of the $\mu^{+}$in the gas positiondependent. The drift velocity vector of $\mu^{+}$in gas, in the presence of electric and magnetic fields, can be written as [7]:

$\mathbf{v}_{D}=\frac{\mu|\mathbf{E}|}{1+\omega^{2} / v^{2}}\left[\hat{\mathbf{E}}+\frac{\omega}{v} \hat{\mathbf{E}} \times \hat{\mathbf{B}}+\frac{\omega^{2}}{v^{2}}(\hat{\mathbf{E}} \cdot \hat{\mathbf{B}}) \hat{\mathbf{B}}\right]$,

where $\mu=e / v m$ is the mobility of the muon, $\hat{\mathbf{E}}$ and $\hat{\mathbf{B}}$ are the unit vectors of the electric and magnetic fields, $\omega=e B / m$ is the cyclotron frequency of the muon and $v$ the average collision frequency of the muon with the He gas atoms. The drift velocity $\mathbf{v}_{D}$ can be made position-dependent by applying a position-dependent electric field $\mathbf{E}$ and/or by making 


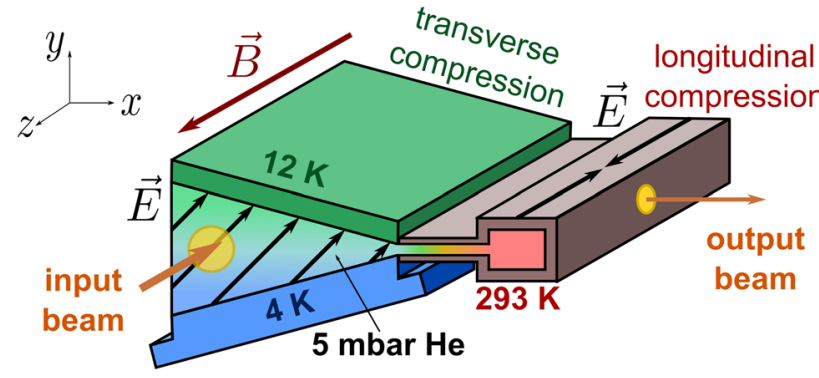

Fig. 1 Overview of the proposed phase space compression scheme. A standard surface muon beam, propagating along the $-z$-direction, is stopped in cryogenic helium gas with a vertical temperature gradient inside a $5 \mathrm{~T}$ magnetic field. The stopped muon swarm is then compressed by means of $\mathbf{E}$ - and $\mathbf{B}$-fields in the $y$-direction ("transverse compression"), and at the same time it is steered in the $x$-direction towards the longitudinal compression stage. There, the He gas is at room temperature, and there is a component of the electric field parallel to the magnetic field axis. Thus, the muon swarm is compressed towards the center of this stage. Finally, the muons with a swarm size of $\mathscr{O}(\mathrm{mm})$ are extracted through a small orifice into vacuum, where they are re-accelerated and sent to the next experiment

the collision frequency $v$ position-dependent, which changes the weights of the three components in Eq. 1.

A sketch of the He gas target where such a compression occurs is shown in Fig. 1, together with the coordinate system used throughout this paper. The target consists of several stages and is placed in a homogeneous magnetic field, oriented along the $+z$-direction, i.e. $\mathbf{B}=(0,0, B)$, with $B=5$ Tesla.

The incoming $\mu^{+}$beam, propagating along the $-z$ direction, is stopped in the first stage which is at cryogenic temperatures, with a vertical ( $y$-direction) gas density gradient induced by a temperature gradient between $T=4 \mathrm{~K}$ at the bottom and $T=12 \mathrm{~K}$ at the top of the roughly $3 \mathrm{~cm}$ high target [8]. The gas density gradient gives rise to a positiondependent collision frequency $v=v(x, y, z)$.

An electric field $\mathbf{E}=\left(E_{x}, E_{y}, 0\right)$ with $E_{x}=E_{y} \approx$ $1 \mathrm{kV} \mathrm{cm}^{-1}$ is applied perpendicular to the magnetic field in this stage, thus only the first two terms in Eq. 1 remain. The muons in a region of higher gas density (colder temperatures) follow the $\hat{\mathbf{E}}$-direction because $v$ is large, whereas muons in a region of lower gas density (warmer temperatures) primarily follow the $\hat{\mathbf{E}} \times \hat{\mathbf{B}}$-direction. With our choice of $\mathbf{E}, \mathbf{B}$ and $v(x, y, z)$ we achieve compression of the stopped muon swarm in the vertical $(y)$ direction (called "transverse compression"), with superimposed drift in the $+x$-direction towards the second compression stage, where longitudinal (in the $z$-direction) compression occurs.

The second stage is at room temperature (much lower gas density) and the electric field is parallel to the magnetic field: $\mathbf{E}=\left(0,0, \mp E_{z}\right)$ pointing towards the center of the target, as indicated in Fig. 1, with $E_{z} \approx 50 \mathrm{~V} \mathrm{~cm}^{-1}$. Therefore, in the second stage Eq. 1 simplifies to $\mathbf{v}_{D} \approx \mu \mathbf{E}$, and the muon swarm is compressed along the $\pm z$-direction ("longitudinal compression") towards $z=0$. Adding an electric field in the $y$-direction gives rise to a superimposed drift in the $+x$ direction, which guides the compressed muon swarm towards an orifice of about $1 \mathrm{~mm}$ diameter, through which the muons are extracted into vacuum. After extraction into the vacuum, the $\mu^{+}$are accelerated to $\mathrm{keV}$ energy, while maintaining eV energy spread, and are subsequently extracted from the magnetic field.

Initially, the various stages of the compression target can be tested separately, simplifying the experimental approach considerably. Transverse compression has been successfully demonstrated and will be presented in a separate publication. Longitudinal compression has been demonstrated to be feasible [9]. However, several problems prevented quantification of the muon swarm compression efficiency. The major issue was impurities in the helium gas target, which captured lowenergy muons and thus lowered the compression efficiency. Moreover, a large background allowed us to demonstrate only qualitatively the feasibility of the longitudinal compression.

In this paper we present an improved demonstration of the longitudinal compression with a quantification of the compression efficiency. Furthermore, we demonstrate that the muon swarm can be steered in the $+x$-direction by adding a vertical ( $y$-direction) electric field. This is crucial to guiding the $\mu^{+}$through the various compression stages and finally towards the point of extraction. The experiments were performed at the $\pi \mathrm{E} 1$ beam line at the Paul Scherrer Institute (PSI), which delivers about $10^{4} \mu^{+} / \mathrm{s}$ at $11 \mathrm{MeV} / \mathrm{c}$.

\section{Test of longitudinal compression}

In order to test longitudinal compression, an $11 \mathrm{MeV} / \mathrm{c}$ muon beam was injected longitudinally into a target containing $\mathrm{He}$ gas of a few mbar pressure at room temperature. The setup is sketched in Fig. 2. The muons first have to pass through a $55 \mu \mathrm{m}$ thick entrance detector (D1), giving the initial time $t_{0}=0$, then through a $2 \mu \mathrm{m}$ Mylar target window enclosing the He gas. Only a small fraction $\mathscr{O}(1 \%)$ of the muons producing the signal in D1 stop in the gas, while the remaining muons stop in D1 or the target window (region I in Fig. 2) or the downstream part of the target (region II in Fig. 2).

The longitudinal compression target had a transverse cross section of $12 \times 12 \mathrm{~mm}^{2}$, and an "active" length, within which the electric field was defined, of about $200 \mathrm{~mm}$. The side walls of the target were lined with gold electrodes that created a $\mathrm{V}$-shaped electric potential with a minimum at the center of the target cell at $z=0$. This choice of the electric potential produced an electric field pointing towards $z=0$, which 


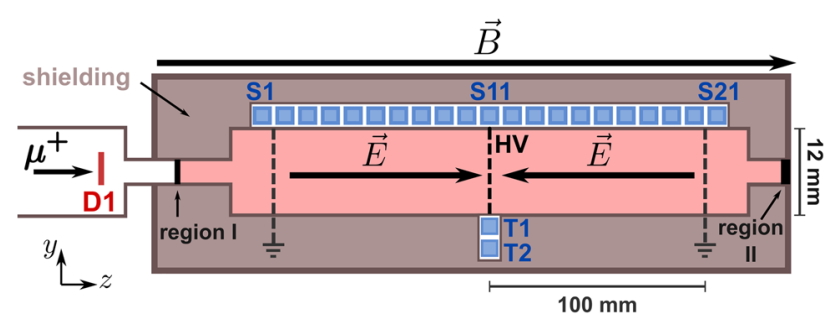

Fig. 2 Sketch of the He gas target used to measure the longitudinal compression (not to scale). The muon beam passes through an entrance detector D1, target window (region I) and enters the He gas (indicated in pink). Some of the muons are stopped in the gas and subjected to the electric field, pointing towards the center of the target. A large fraction of the muons pass through the target and stop in region II. Several scintillators $(\mathrm{S} 1, \ldots, \mathrm{S} 21, \mathrm{~T} 1, \mathrm{~T} 2)$ detect the positrons from muons decaying in their vicinity

caused the muons to move along the $\pm z$-direction towards the potential minimum. ${ }^{1}$

The exact electric field was simulated using a finiteelement method within COMSOL Multiphysics® [10] and imported into the Geant4 simulation package [11], allowing us to study muon motion in the realistic electric field. The Geant4 simulation included the most relevant low-energy processes down to $\mathrm{eV}$ energies, namely low-energy elastic collisions $\left(\mu^{+}-\mathrm{He}\right.$ and muonium $\left.-\mathrm{He}\right)$ and charge exchange processes (muonium formation and ionization) [9]. Cross sections for elastic collisions and charge exchange were implemented by energy and velocity scaling [12], respectively, of the proton cross sections from $[13,14]$.

A simulation of the muon distribution inside the target at two different times $t$ is shown in Fig. 3. The distribution at $t=0.15 \mu$ s represents the initial muon stop distribution, while the distribution at $t=2 \mu \mathrm{s}$ shows the muon distribution when the longitudinal compression is almost completed. To highlight the muon swarm compression, the number of counts has been scaled with $\exp (t / \tau)$, where $\tau=2197 \mathrm{~ns}$ is the muon lifetime, in order to compensate for muon decay.

Under such conditions and neglecting muon decay, at $t=$ $2 \mu \mathrm{s}, 63 \%$ of the muons that were in the active region at $t=0.15 \mu \mathrm{s}$ are still within the active region, with $90 \%$ of those already within $z= \pm 5 \mathrm{~mm}$. Moreover, $50 \%$ of all muons in the active region at $t=2 \mu \mathrm{s}$ are already compressed in the center within an even smaller region of $z= \pm 1 \mathrm{~mm}$. Therefore, the fraction of muons within the region of $z=$ $\pm 1 \mathrm{~mm}$ increases by about a factor of 20 between $0.15 \mu \mathrm{s}$ and $2 \mu \mathrm{s}$.

The other $37 \%$ of the muons that were initially in the active region are lost through two main mechanisms: $26 \%$ through muonium formation and $11 \%$ through scattering out of the

\footnotetext{
${ }^{1}$ Because of $\nabla \cdot E=0$, at $z=0$ the electric field will also have a radial component. This component is included automatically in the COMSOL Multiphysics ${ }^{\circledR}$ simulation but it is so small that it does not significantly affect the muon motion.
}

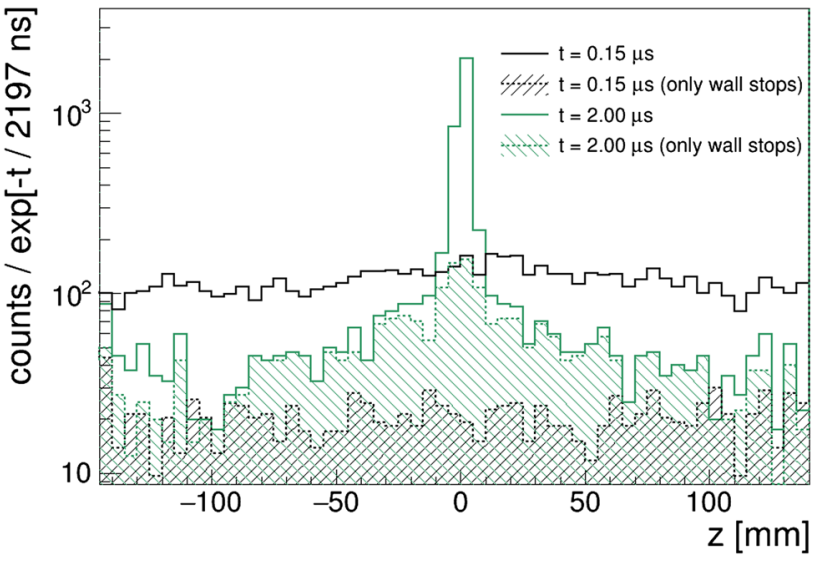

Fig. 3 Simulated muon distribution along the $z$-axis for various times. The histograms have been corrected for the finite muon lifetime by multiplying the counts with $e^{t / 2197 \mathrm{~ns}}$. As can be seen, for $t=2 \mu \mathrm{s}$ most of the muons are close to the center of the active region. The shaded areas show the fraction of the $\mu^{+}$that stopped in the walls of the target. At $t=2 \mu \mathrm{s}$, the distribution of the $\mu^{+}$adhering to the target walls is not flat anymore. The reason for the peak around $z=0$ is that some of the $\mu^{+}$are scattered into the wall while drifting towards the center of the target

active region due to low-energy elastic collisions. A muon bound in the neutral muonium atom is not confined by the 5 tesla magnetic field, causing it to fly into the walls of the target. The losses due to scattering of $\mu^{+}$are relevant only when the muon beam is injected into the longitudinal target at $\mathrm{keV}$ energies, because the $\mu^{+}$mean free path at these energies can be up to a few $\mathrm{cm}$. These losses will therefore be absent in the final setup, in which muons enter the longitudinal compression stage from the transverse compression stage at $\mathrm{eV}$ energies. Indeed, at such energies, the $\mu^{+}$mean free path is sub-mm. Similarly, the losses due to muonium formation will be strongly reduced in the final setup.

The muon swarm movement was measured experimentally by placing 21 identical plastic (BC-404) scintillators (S1 to S21) along the target $z$-axis, as shown in Fig. 2. The scintillators had dimensions of $30 \times 5 \times 6 \mathrm{~mm}^{3}$ and were wrapped in Teflon ${ }^{\mathrm{TM}}$ tape. When positrons from muon decays pass through the scintillators they produce scintillation light which is then read out by Silicon Photomultipliers (SiPMs) with $3 \times 3 \mathrm{~mm}^{3}$ active area. The acceptance of some of these scintillators as a function of muon decay $z$-position is plotted in Fig. 4. Each detector has an average geometrical acceptance in the $z$-direction of $16.5 \mathrm{~mm}$ (FWHM).

The number of measured positrons in each scintillator is presented for $t=150 \mathrm{~ns}$ (black dots) and $t=2000 \mathrm{~ns}$ (green dots) in Fig. 5. Note that the number of counts has been scaled with $e^{t / 2197 \text { ns }}$ to compensate for muon decay.

In Fig. 5 the measured $\mu^{+}$distributions (dots) are compared to the Geant4 simulations (curves). The simulated number of positron hits in each of the detectors S1-S21 


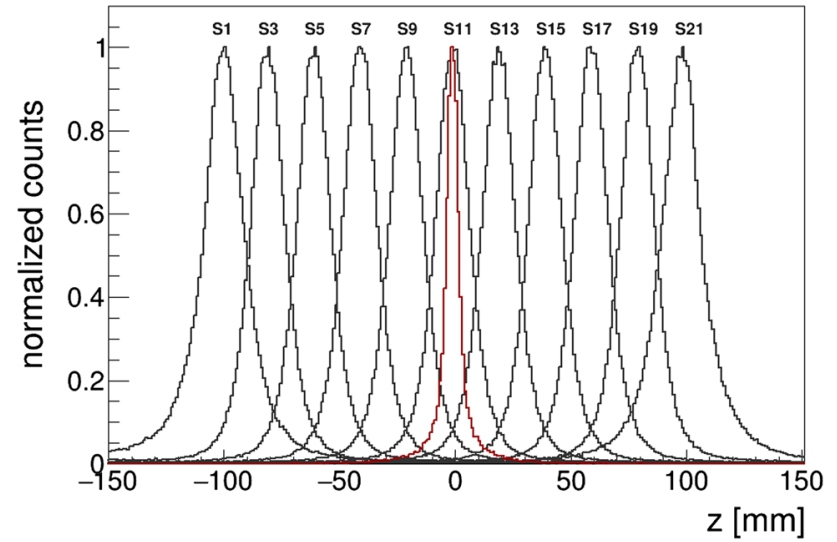

Fig. 4 Simulated geometrical acceptance of scintillators S1-S21 (only every second detector is shown) versus $z$-position. Shown is the normalized probability that a positron from a $\mu^{+}$decay will be detected in the corresponding scintillator. For comparison, the geometrical acceptance of the T1 \& T2 coincidence is also plotted (red line). The maximum of the efficiency for each detector was normalized to 1 to highlight the different geometrical resolutions

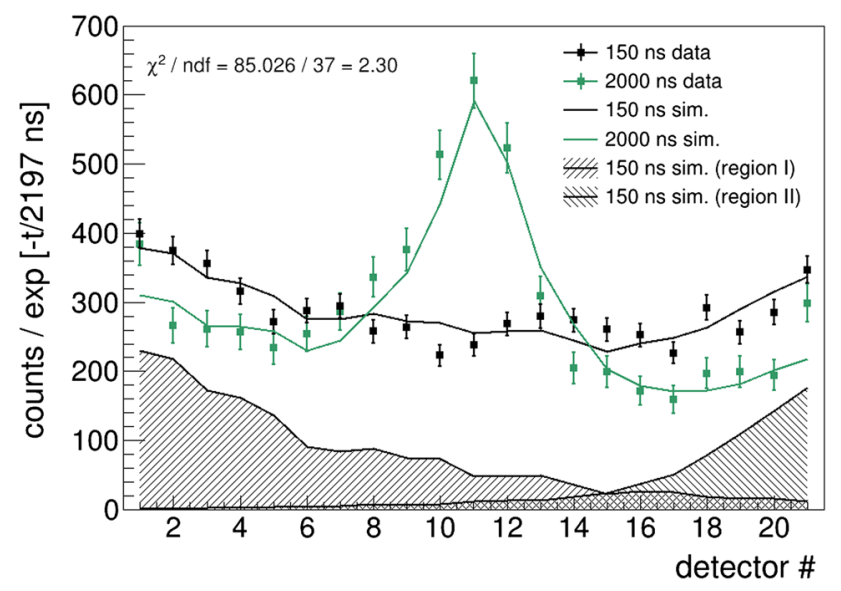

Fig. 5 Measured (dots) and simulated (curves) positron hits in the 21 scintillators shown in Fig. 2, for $t=150 \mathrm{~ns}$ and $t=2000 \mathrm{~ns}$. The center-to-center distance between two adjacent scintillators is $10 \mathrm{~mm}$. The simulated data are the same as in Fig. 3, but convoluted with the geometrical acceptance of the detectors. The width of the peak at $t=$ $2 \mu \mathrm{s}$ is due to the large geometrical acceptance of the 21 scintillators and does not reflect the width of the muon swarm directly (compare with Fig. 3). The shaded areas show the simulated contributions to the positron hits from muons stopped in regions I and II. The data and the simulations have been corrected for the finite muon lifetime by multiplying the counts by $e^{t / 2197 \mathrm{~ns}}$

is obtained by convoluting the simulated spatial distribution of the muon swarm at the corresponding time, given in Fig. 3, with the detection efficiency of the appropriate detector, given in Fig. 4. These positron hits originate not only from the muons in the active region, but also from muons from the regions where the electric field is not well defined, giving rise to a substantial background.

This background is dominated by positron hits from $\mu^{+}$ that stop in the regions I and II (as defined in Fig. 2). The background is larger for the detectors placed at the periphery of the active region (S1, S2 and S20,S21), as shown in Fig. 5 (shaded areas). The shape of the background caused by these muons can be simulated. However, the exact number of $\mu^{+}$ which stop in the regions I and II depends strongly on the momentum distribution of the initial muon beam, which is not sufficiently well known.

Therefore, two measured distributions along the $z$-axis (for $t=150 \mathrm{~ns}$ and $t=2000 \mathrm{ns)}$ ) are fitted simultaneously with the sum of 4 contributions:

1. Background arising from the region I.

2. Background arising from the region II.

3. Linear (in time) background.

4. Simulation of all $\mu^{+}$that stop in the gas (which includes $\mu^{+}$in the active region).

The shapes of these 4 contributions are known, under the assumption that all the detectors (S1 to S21) have the same detection efficiency. Each of the contributions has to be scaled with a different scaling factor to account for the different stopping probabilities in region I, region II, the gas, and prompt stops at the target lateral walls. The additional linear background allows us to account for possible misalignment between the target and the magnetic field axis, which would lead to different numbers of muon wall stops at the positions of the various scintillators.

Even though we observe fair agreement between the measurement and the simulation (reduced chi-square ${ }^{2} \chi_{\text {red }}^{2}=$ 2.3 , for 37 degrees of freedom), it is difficult to extract precise values of the compression efficiency and of the width of the muon swarm from these measurements, given the limited geometrical resolution of the detectors $\mathrm{S} 1$ to $\mathrm{S} 21$ and the large background from regions I and II. The relatively large $\chi_{\text {red }}^{2}$ could be attributed to small variations of the detector efficiencies.

In order to better quantify the compression efficiency we turn our attention to the two telescope detectors T1 \& T2 in coincidence that were placed below the target, at $z=0$, as shown in Fig. 2. These scintillators had dimensions of $32 \times 3 \times 3 \mathrm{~mm}^{3}$ and were also read out by $3 \times 3 \mathrm{~mm}^{2}$ SiPMs. Massive brass shielding all around the target ensured that

${ }^{2}$ In this paper we are using following definition of the reduced chisquare:

$\chi_{\text {red }}^{2}=\frac{1}{\operatorname{ndf}} \sum_{i=1}^{N} \frac{\left(n_{\text {data }, i}-n_{\mathrm{sim}, i}\right)^{2}}{\sigma_{\text {data }, i}^{2}}$,

where $N$ is the number of bins in the histogram, $n_{\mathrm{data}, i}$ and $n_{\mathrm{sim}, i}$ are the numbers of counts in the $i$-th bin of the data and simulation histograms, respectively, $\sigma_{\mathrm{data}, i}$ is the statistical uncertainty of $n_{\mathrm{data}, i}$ and ndf is the number of degrees of freedom (equal to $N$ - the number of fitted parameters). The statistical uncertainty of the simulated points is significantly smaller than $\sigma_{\text {data }, i}$, and can therefore be neglected. 


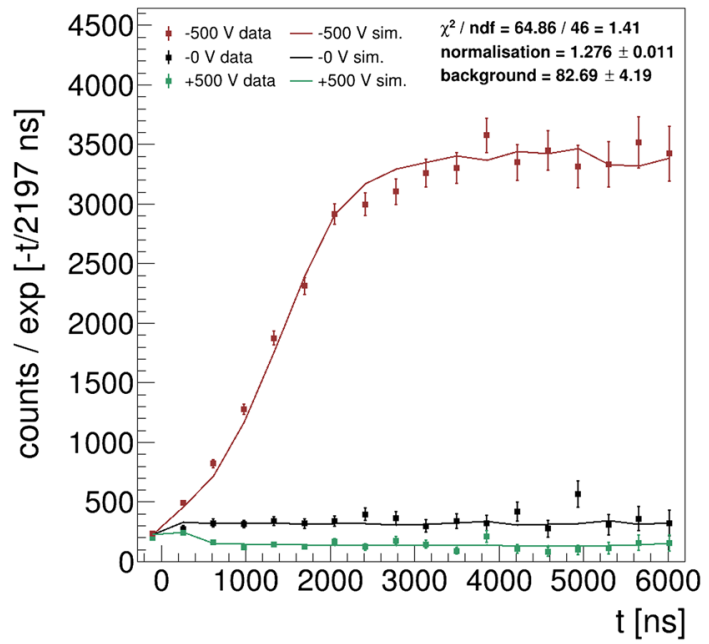

Fig. 6 Measured (dots) and simulated (curves) time spectra for 5 mbar $\mathrm{He}$ gas pressure and potentials of $-500 \mathrm{~V}$ (red), $0 \mathrm{~V}$ (black) and $+500 \mathrm{~V}$ (green). The data have been normalized to the number of incoming muons in the entrance detector D1 and fitted simultaneously with only 2 free parameters, a common normalization and a common background. In total $5 \cdot 10^{8}$ muons have been simulated. The data and the simulations have been corrected for the finite muon lifetime by multiplying the counts by $e^{t / 2197 \mathrm{~ns}}$

coincidence hits in $\mathrm{T} 1 \& \mathrm{~T} 2$ originated only from muons decaying within the small region between about $z= \pm 3 \mathrm{~mm}$ in the center of the target, as shown in Fig. 4. From the time difference $t=t_{1}-t_{0}$ between the positron hits in T1 and $\mathrm{T} 2$ in coincidence (at time $t_{1}$ ) and the entrance detector, at time $t_{0}$, a time spectrum can be obtained as shown in Fig. 6. The time spectra were recorded for different applied electric potentials.

It can be seen in Fig. 6 that if no electric field is applied (black points) the number of muons decaying in the center of the target (in the T1 \& T2 acceptance region) stays constant (after compensation for $\mu^{+}$decay). When a negative potential (red points) is applied in the center of the target cell, the measured number of counts increases with time. In that case, the stopped muons are attracted towards the potential minimum, so that more muons decay within the acceptance region of $\mathrm{T} 1 \& \mathrm{~T} 2$. This means that the muon swarm extent has been decreased in the $z$-direction, representing a complementary way to demonstrate longitudinal compression. On the contrary, if the "wrong" polarity is applied (green points), the muons drift away from the center of the target, out of the acceptance region of $\mathrm{T} 1 \& \mathrm{~T} 2$. The very few counts at late times in that case are due to some small background (mostly muons stopping in the wall of the target). The reduction to a nearly background-free measurement represents a major improvement compared with the earlier measurements from 2011 [9] and compared to the measurement of the $\mu^{+}$ $z$-distribution of Fig. 5.
The measurements of Fig. 6 are compared to Geant 4 simulations. The three simulations $(+,-$ and 0 voltage) are fitted simultaneously to the corresponding measured time spectra. Only two free parameters were used: a common scaling factor and a common flat background. The common scaling factor is needed to remove the uncertainties related to the positron detection and muon stopping efficiencies. The flat background was included in the fit to account for potential misalignment of the target with respect to the magnetic field axis, which would lead to increased muon stops in the walls.

The simultaneous fit of the 3 curves has a reduced chisquare $\chi_{\text {red }}^{2}=1.41$ (for 46 degrees of freedom). Introduction of additional losses during the compression process, as detailed in the next section, improves the $\chi_{\text {red }}^{2}$ value to 0.95 . Alternatively, a smaller $\chi_{\text {red }}^{2}$ can also be obtained by a minor tuning of the detector acceptance, related to uncertainties in the position, tilt and energy threshold of the T1 \& T2 scintillators.

\section{Additional muon losses?}

As mentioned in the introduction, in the previous (2011) experiment [9], the data displayed lower-than-expected compression efficiency, which was visible from the premature termination (at around $t=0.5 \mu \mathrm{s}$ ) of the compression in the measured time spectra. This was attributed to severe losses of "free" muons, hypothesized to have been caused by impurities present in the helium gas (from outgassing of the Araldite glue and PCB boards used in the target construction). The presence of such impurities would cause the capture of lowenergy muons by the contaminant molecules. Additionally, other issues affected the previous measurements such as misalignment between target and magnetic field axis, small target size and poor spatial resolution of the positron detector. These issues suppress the rise in the time spectra, thereby imitating the signature of the "free" muon losses, making it difficult to quantify the actual compression efficiency.

In the experiments presented here, care was taken to improve the aspects limiting the previous experiment: the detector resolution was enhanced by introducing more shielding around the positron detectors and using the telescope detectors (T1 \& T2) in coincidence instead of a single detector, the target size was increased and the electric field strength and homogeneity were improved. As a consequence, the sensitivity to target misalignment was considerably reduced. Additionally, high gas purity was ensured by constructing the target from glass plates, using a lowoutgassing glue and purifying the He gas in a cold-trap before feeding it into the target.

In order to prove the hypothesis that indeed the compression efficiency is lowered if contaminants are present in the gas, we introduced in a controlled way various amounts of 


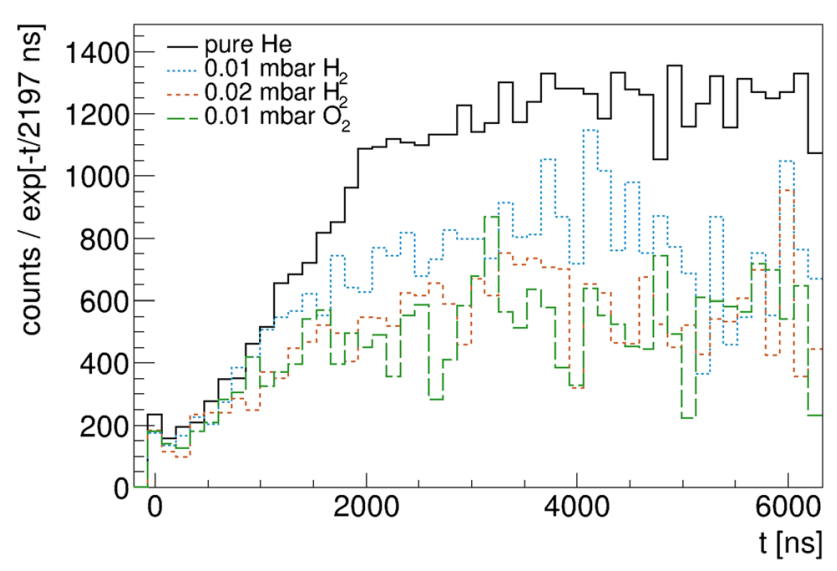

Fig. 7 Measured time spectra for 5 mbar pure He gas, and various admixtures of contaminants: (black) no additional contaminants, (dashed blue) $0.01 \mathrm{mbar}_{2}$, (dashed orange) $0.02 \mathrm{mbar}_{2}$, (dashed green) $0.01 \mathrm{mbar}$ of $\mathrm{O}_{2}$. All data were normalized to the number of incoming muons and corrected for the finite muon lifetime by multiplying the counts bt $e^{t / 2197 \mathrm{~ns}}$

contaminant gases into the pure helium gas. The results of this test are presented in the time spectra of Fig. 7. It is observed that the compression stops at earlier times when contaminants are introduced, and consequently the compression efficiency decreases (i.e., the number of muons that are brought to the center of target).

The sensitivity of the measurements of Fig. 6 to the muon loss mechanisms has been investigated assuming constant (in time) effective loss rates $R$ during compression, that cause the "free" muon population to decrease according to $e^{-R t}$. Various simulations have been performed with $R$ ranging from $R=0$ up to $R=0.5 \mu \mathrm{s}^{-1}$. The time spectrum with an effective loss rate $R=0.5 \mu^{-1}$ would correspond roughly to the measured time spectrum with $0.01 \mathrm{mbar}_{2} \mathrm{H}_{2}$ contamination (blue dashed line in Fig. 7).

The simultaneous fit of the simulations for,+- and 0 voltages to the corresponding measurements has been performed for each effective loss rate $R$ (as in Fig. 6, but for $R \neq 0$ ). Figure 8 (top) shows the fitted time spectra (only for negative voltage) for several effective loss rates $R$. For each of the effective loss rates $R$, the $\chi_{\text {red }}^{2}$ between the measurement (only $-500 \mathrm{~V}$ data) and simulations has been calculated and plotted in Fig. 8 (bottom). A parabola was then fitted to these points (green line). The best agreement (minimum $\chi_{\text {red }}^{2}$ ) between simulation and measurement is obtained for $R=0.14 \mu^{-1}$, corresponding to an additional effective loss at $t=2 \mu \mathrm{s}$ of $1-e^{-0.14 \mu \mathrm{s}^{-1} \cdot 2 \mu \mathrm{s}}=24 \%$. The $\chi_{\text {red,min }}^{2}+1$ is obtained for $R=0.35 \mu \mathrm{s}^{-1}$ corresponding to an additional loss at $t=2 \mu$ s of $51 \%$. Therefore, we conclude that the total additional "free" muon loss after $2 \mu \mathrm{s}$ is $24_{-24}^{+27} \%$.

Had these losses been caused by capture of the muons by gas impurities, the partial pressure of the impurities would
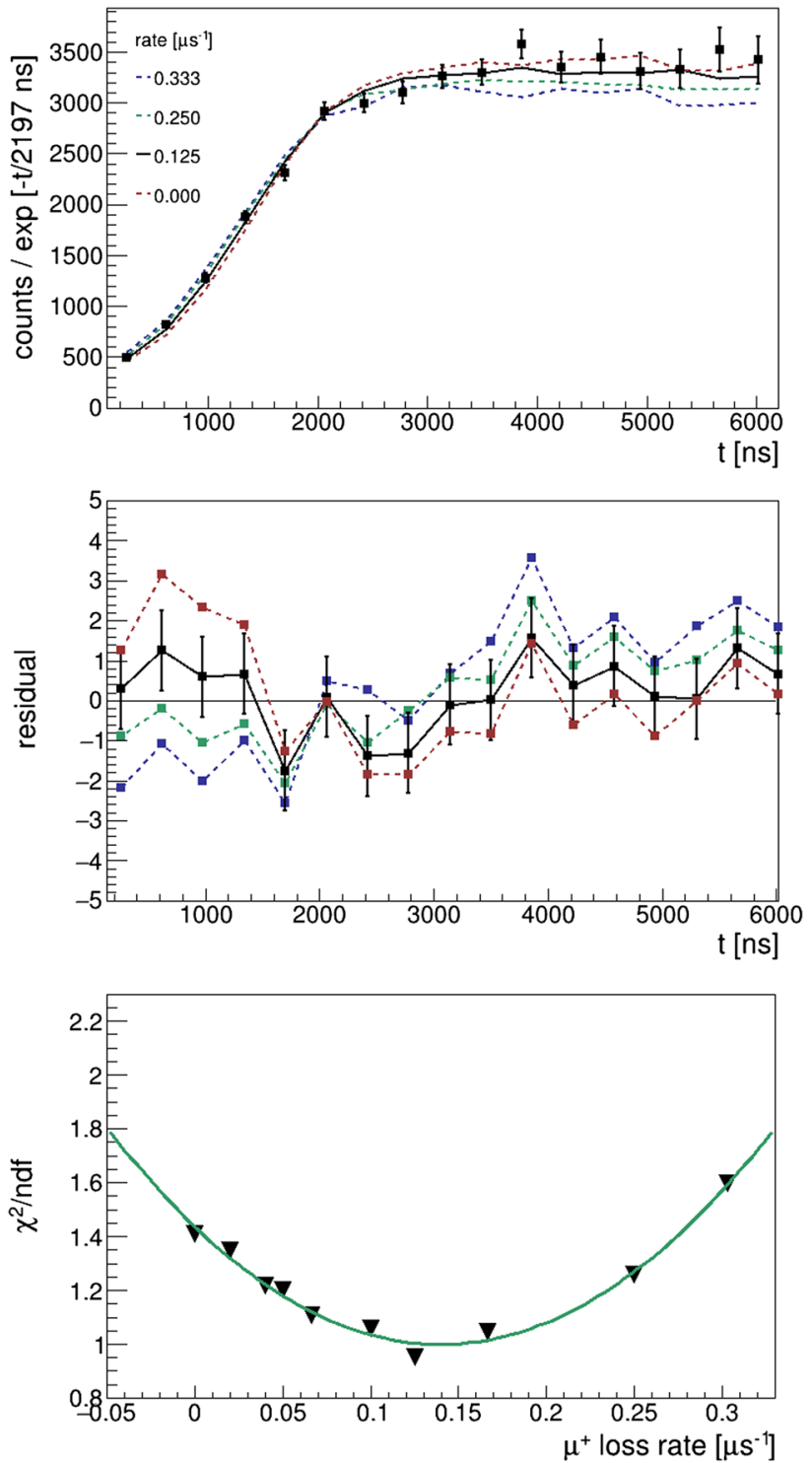

Fig. 8 (Top) Simulated time spectra for effective loss rates of $R=$ $(0,0.125,0.25,0.333) \mu \mathrm{s}^{-1}$ (curves). The $-500 \mathrm{~V}$ measurement is represented by square points. The data and the simulations have been corrected for the finite muon lifetime by multiplying the counts by $e^{t / 2197 \mathrm{~ns}}$. (Middle) Residuals for the time spectra from the top figure. For simplicity, error bars are shown only for the curve with reduced $\chi^{2}=0.95$. For other curves error bars are of similar size. (Bottom) Reduced $\chi^{2}$ as a function of the effective loss rate $R$. A second-order polynomial was fitted to these points (green line)

have been $\sim 10^{-3}$ mbar. $^{3}$ However, the purity of the He gas in the target was analyzed with a mass spectrometer and such impurity levels have not been observed. Thus, it is reasonable to conclude that the origin of these "effective losses" $R$ cannot be attributed solely to the impurities.

\footnotetext{
3 The relation between fitted $R$ and impurity concentration is obtained by fitting the simulation for various loss rates $R$ to the measured time spectra of Fig. 7 with the additional $0.01 \mathrm{mbar}_{2}$ contamination.
} 
Other explanations, such as small deviations of the detector acceptance (spatial resolution) and of the cross sections from the values implemented in the simulations, are more favored. For example, a shift of only $0.5 \mathrm{~mm}$ (which is within the tolerance of the mechanics) of the T1 $y$-position from the design position would decrease the acceptance of $\mathrm{T} 1 \&$ $\mathrm{T} 2$ in coincidence by $5 \%$. This would then translate to a $5 \%$ decrease of the number of counts at late times in the measured time spectra of Fig. 8 (top), thereby imitating the signature of the "free" muon loss. This 5\% decrease in the number of counts at late times roughly corresponds to the decrease caused by an effective loss rate $R=0.125 \mu \mathrm{s}^{-1}$ (compare red and black lines of Fig. 8 (top)).

\section{E $\times$ B-Drift}

The next important step towards the realization of the complete muCool device is the demonstration of the so-called $\mathbf{E} \times \mathbf{B}$ drift. This drift guides the muons from one compression stage to the next, and is also used to extract them finally into vacuum through a small orifice.

For this purpose we modified the target cell to generate an electric field with an additional vertical component $E_{y}=120 \mathrm{~V} / \mathrm{cm}$. The non-vanishing central term in Eq. 1 leads to a drift of the muons in the $+x$-direction. The target cell was enlarged to a transverse cross section of $24 \times 12 \mathrm{~mm}^{2}$ and, additionally, the muon beam was injected off-center, as indicated in Fig. 9 by the yellow circle. These two modifications allowed $\mu^{+}$to drift for longer times before hitting the right wall of the target, thus enhancing the sensitivity of the measured time spectra to the muon drift.

In total seven plastic scintillators were mounted around the target at $z=0$ along the $x$-direction to monitor this drift, as shown in the sketch of Fig. 9. For simplicity, we present only the time spectra from three of them, namely "Left", "Middle", "Right", given their favorable detection efficiency and sensitivity to the muon motion. As before, the scintillators were read out by $3 \times 3 \mathrm{~mm}^{2}$ SiPMs.

The simulated spatial distribution of the muon swarm as a function of time is given in Fig. 10. This distribution, convoluted with the corresponding detector acceptances, gives rise to the simulated time spectra shown in Fig. 11, together with the corresponding measurements.

At early times, the time spectra are dominated by the muon swarm compression in the $z$-direction, thus the number of detected positrons increases in all scintillators. However, after about $2 \mu \mathrm{s}$, the number of detected positrons in the "Left" scintillator decreases, indicating that the muons are moving out of the acceptance region of this scintillator. On the other hand, "Right" scintillator detects increasingly more positrons. This finding indicates that the muon swarm

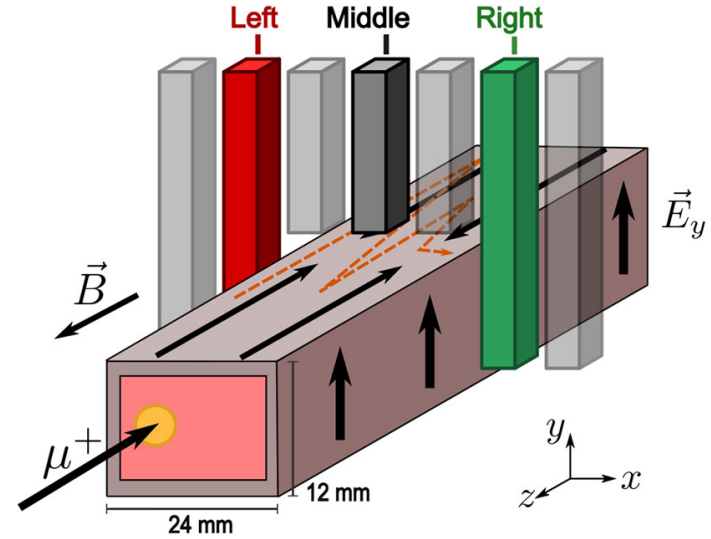

Fig. 9 Sketch of the setup to measure the $\mathbf{E} \times \mathbf{B}$ drift. The muon beam was injected off-axis to allow the muons to drift a longer distance before hitting the lateral wall. The electric field has a $z$-component for longitudinal compression and a $y$-component to drift the muons in $x$-direction. Seven plastic scintillators are positioned above the target at $z=0$, but at different $x$-coordinates, to monitor the muon swarm movement in the $x$-direction. The scintillators had a cross section of $2 \times 2 \mathrm{~mm}^{2}$ and length (in the $y$-direction) of $32.5 \mathrm{~mm}$ for the three central scintillators and $48.5 \mathrm{~mm}$ for the four scintillators on the left and right sides of the target

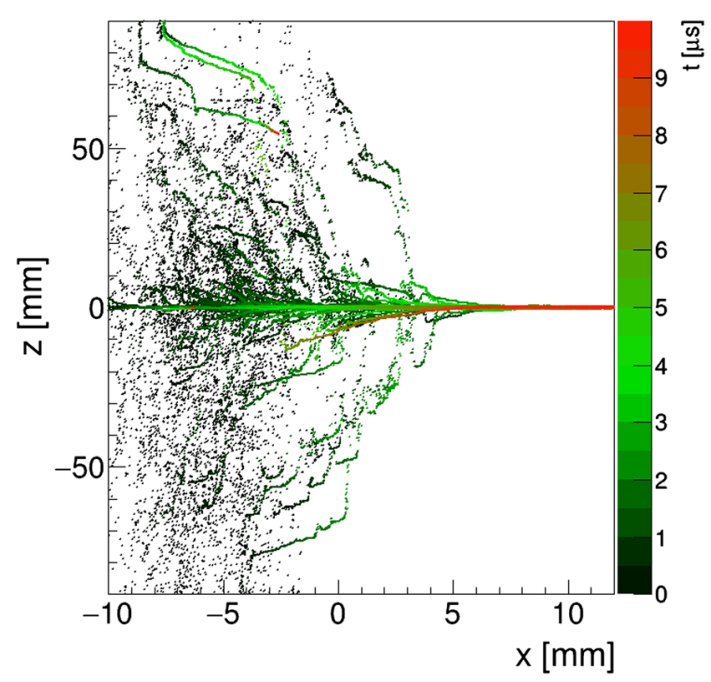

Fig. 10 Muon positions in the $x z$-plane for various times. The time is given by the color scale. Muon beam centered at $x=-6 \mathrm{~mm}$ with $3 \mathrm{~mm}$ radius is stopped uniformly along the $z$-axis. The muons drift in the $+x$-direction while compression occurs in $z$-direction

slowly drifts in the $x$-direction towards the prospective point of extraction.

Also in this case, the measured time spectra of all seven "drift" detectors were fitted simultaneously with the simulation allowing for one common scaling factor, and a different flat background for each detector. A fair agreement between data and simulation has been observed $\left(\chi_{\text {red }}^{2}=3.44\right.$ for 433 degrees of freedom), given that no systematic effects were accounted for in the simulation. 


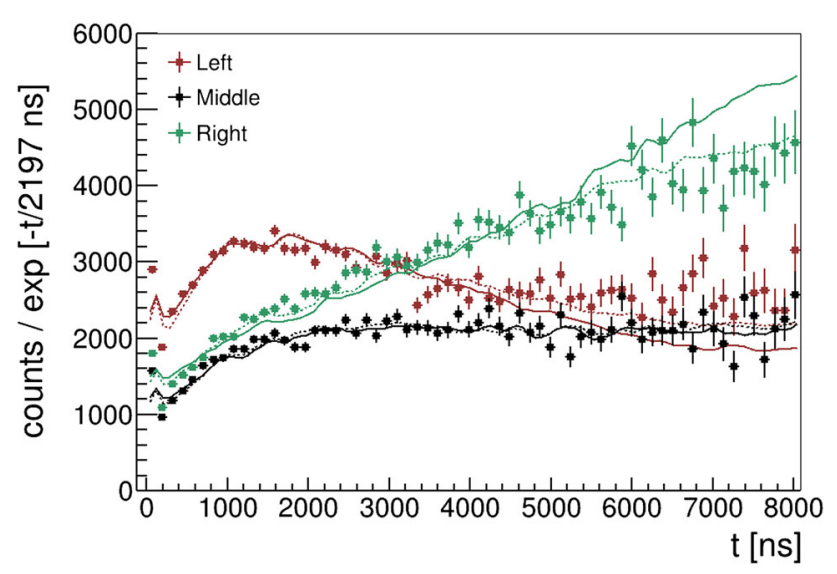

Fig. 11 Measured (dots) time spectra for the three scintillators "Left", "Middle" and "Right". The increase and decrease of positron counts in the "Right" and "Left" detectors, respectively, indicates that the muons are moving towards the right. Simulated time spectra for additional muon loss rate $R=0$ (continuous lines) and $R=0.125 \mu \mathrm{s}^{-1}$ (dashed lines) are fitted to the measured time spectra. The $\chi_{\text {red }}^{2}$ for $R=0$ is 3.44 for $433^{\circ}$ degrees of freedom. Introducing the additional loss rate $R=0.125 \mu \mathrm{s}^{-1}$ improves the $\chi_{\text {red }}^{2}$ to 2.23 . Note that the data and the simulations have been corrected for the finite muon lifetime by multiplying the counts with $e^{t / 2197 \mathrm{~ns}}$

To study the effect of additional muon losses on the measured time spectra, an effective loss rate $R$ has been introduced in the simulation, analogously to the procedure described in the previous section. The obtained time spectra for the various $R$ were then fitted to the data. The best agreement between simulation and the data is obtained for $R=0.125 \mu \mathrm{s}^{-1}$, consistent with the loss rate $R$ reported in the previous section. The best fit, which gives a $\chi_{\text {red }}^{2}=2.23$, is shown in Fig. 11 (dashed lines).

To extract the uncertainty of the hypothetical loss rate that describes the data the best, we repeated the procedure of the previous section: namely, fitting with a parabola the plot of the reduced chi-square versus loss rate (similar to Fig. 8). Note that for that procedure, the error bars of the data were scaled up by a factor of 2.23 to produce a minimum reduced chi-square of 1 . From this analysis we determined that the loss rate that best describes the data is $R=0.17_{-0.17}^{+0.23} \mu \mathrm{s}^{-1}$ corresponding to a total "free" muon loss at $t=2 \mu \mathrm{s}$ of $288_{-28}^{+26} \%$.

Even with the additional muon losses introduced, some systematic discrepancies between the data and the simulation still remain. Yet the main goal, namely to demonstrate the feasibility of the $\mathbf{E} \times \mathbf{B}$ drift, has been achieved.

The difference between the simulation and the measurement can be attributed either to the simplified modelling of the additional losses (without any energy dependence) or a misalignment of the beam with respect to the magnetic field axis.
According to the simulation, the drift velocity is about $2 \mathrm{~mm} / \mu \mathrm{s}$. This value can be increased in the final setup by increasing the strength of the electric field in the $y$-direction.

\section{Conclusions}

The longitudinal compression stage of the muCool device under development at PSI has been demonstrated. An elongated muon swarm of $200 \mathrm{~mm}$ length has been compressed to below $2 \mathrm{~mm}$ length within $2 \mu \mathrm{s}$. Good agreement between the simulation and the measurement has been observed.

Furthermore, the ability to drift the $\mu^{+}$beam in $\mathbf{E} \times \mathbf{B}$ direction towards the prospective position of the extraction hole has been demonstrated by performing a measurement with the electric field having also a component perpendicular to the magnetic field.

In both cases, slightly better agreement between simulations and measurements is achieved by including small additional effective losses in the simulation, that decrease the actual compression efficiency by about $25 \%$ relative to (ideal) simulations. These effective losses could be caused by gas impurities. Alternatively, the small discrepancies between the simulations and measurements could be attributed to a minor $(5 \%)$ overestimation of the detector acceptance or minor variation of the cross sections for muonium formation, muonium ionization and muonium-He elastic scattering. In any case, even with this additional effective loss, the proposed $\mu^{+}$compression efficiency of $10^{-3}$ is attainable.

Acknowledgements The experimental work was performed at the proton accelerator at PSI. We thank the machine and beam line groups for providing excellent conditions. We gratefully acknowledge the outstanding support received from the workshops and support groups at ETH Zurich and PSI. Furthermore, we thank F. Kottmann, M. Horisberger, U. Greuter, R. Scheuermann, T. Prokscha, D. Reggiani, K. Deiters, T. Rauber, and F. Barchetti for their help. This work was supported by the SNF Grants No. 200020_159754 and 200020_172639.

Data Availability Statement This manuscript has no associated data or the data will not be deposited. [Authors' comment: Raw or preprocessed data and analysis software used can be provided by the corresponding author upon request.]

Open Access This article is distributed under the terms of the Creative Commons Attribution 4.0 International License (http://creativecomm ons.org/licenses/by/4.0/), which permits unrestricted use, distribution, and reproduction in any medium, provided you give appropriate credit to the original author(s) and the source, provide a link to the Creative Commons license, and indicate if changes were made. Funded by $\mathrm{SCOAP}^{3}$.

\section{References}

1. S. van der Meer, Rev. Mod. Phys. 57, 3 (1985)

2. G.I. Budker, A.N. Skrinskii, Sov. Phys. Usp. 21, 277 (1978)

3. M. Mühlbauer et al., Hyperfine Interact. 119, 1-4 (1999) 
4. T. A. Mohayai [MICE Collaboration]. arXiv:1806.01807

5. P. Bakule, E. Morenzoni, Contemp. Phys. 45, 3 (2004)

6. D. Taqqu, Phys. Rev. Lett. 97, 194801 (2006)

7. W. Blum, W. Riegler, L. Rolandi, Particle detection with drift chambers, 2nd edn. (Springer, Berlin, 2008)

8. G. Wichmann et al., Nucl. Instrum. Methods A 814, 33-38 (2016)

9. Y. Bao et al., Phys. Rev. Lett. 112, 224801 (2014)
10. COMSOL Multiphysics®5.2. http://www.comsol.com. COMSOL $\mathrm{AB}$, Stockholm, Sweden

11. S. Agostinelli et al., Nucl. Instrum. Methods A 506, 3 (2003)

12. M. Senba, J. Phys. B 22, 12 (1989)

13. P.S. Krstic, D.R. Schultz, Phys. Plasmas 13, 5 (2006)

14. Y. Nakai et al., At. Data Nucl. Data Tables 37, 1 (1987) 